\title{
Improving Task Scheduling in Large Scale Cloud Computing Environment using Artificial Bee Colony Algorithm
}

\author{
R.Sathish Kumar \\ Assistant Professor, CSE \\ Coimbatore Institute Of \\ Engineering and Technology, \\ Narasipuram, Coimbatore.
}

\author{
S.Gunasekaran, PhD \\ Professor and Head, CSE \\ Coimbatore Institute Of \\ Engineering and Technology \\ Narasipuram, Coimbatore.
}

\begin{abstract}
In the face of Scheduling, the tasks are scheduled by using Different scheduling Algorithms. Each Scheduling Algorithm has own particularity and complexity during Scheduling. In order to get the minimum time for the execution of the task the Scheduling algorithm must be good, once the performance of the scheduling algorithm is good then automatically the result obtained by that particular algorithm will be considered , there are huge number of task that are scheduled under cloud computing in order to get the minimum time and the maximum through put the Scheduling algorithm plays an important factor Here the algorithm which used for Scheduling the task is artificial bee colony algorithm this scheduling process is done under the cloud computing environment. In this Paper we are considering the time as the main QoS factor, minimum total task finishing time, mean task finishing time and load balancing time is obtained by using this Cloud simulation environment
\end{abstract}

Keywords: Scheduling, Complexity, Performance, Cloud Computing, Total task finishing time, Mean task finishing time, Load balancing time, Quality of Services

\section{INTRODUCTION}

The cloud computing is a recent emerging field in the Computational and scheduling techniques, which aims at the scheduling the task must be very scalable and virtualized resources over the internet here it is defined as a distributed system containing a collection of computing and communicating resources located in the distributed datacenter which are shared by several end users cloud computing allow users to run application remotely including information technology services called Software-as-a-services and Infrastructure-as-a-Services by using this services the application is carried out via useful function and services provided by cloud. Between Saas and Iaas there are cloud platform services known as Platform-as-a-Services deliver operating system, programming language and the solution stack. It aims to improve the application deployment cost reduce the complexity. It can be seen that the cloud resource can be simplified and efficiency operated by the scheduling services use in optimal manner in the interest of end user. An algorithm under working it is divided into two groups they are evolutionary algorithm and swarm Intelligence based algorithm. The most popular Among the evolutionary algorithm is Genetic algorithm in this genetic algorithm attempts to simulate the phenomenon of natural evolution. A branch of nature inspired is called swarm intelligence and this focus on some collective behavior of self-organized system the characteristics of swarms inspired huge number of researchers to implement such behavior in computer software for many scheduling problem it is required to apply a special Scheduling algorithm that guarantees the distribution of the workload across thousand datacenters. In the case of load balancing allocation of jobs to datacenter that yields an equal or approximately equal expected response time for all the jobs. To take up the challenge we propose the efficient algorithm called artificial bee colony algorithm inspired by bee's it aims at an optimal job scheduling by assigning end user task to relevant datacenters in optimal way

\section{RELATED WORK}

The most important factor in cloud computing is task scheduling process, in last few years, the task scheduling problem under the cloud computing has been studied in few research activities because of the novelty of the research discipline however; in this paper we mention the following approaches As related work we mentioned the proposed System contains a new Scheduling algorithm which developed for efficient task scheduling process in cloud computing. It provides an efficient Task finishing time, Mean task finishing time and load balancing time. This can be evaluated by the simulation process; the result obtained by this Simulation is very effective and efficient

\section{ARTIFICIAL BEE COLONY SCHEDULING IN CLOUD ENVIRONMENT}

Cloud environment is created by using cloudsim simulation tool. Under cloudsim there are many packages. Source Package is the main package where all the java files are put together. In that package the main concepts are Datacenter, scheduler, virtual machine cloudlets and their corresponding policy's. By using this concept we can implement the ABC Scheduling algorithm in the cloudsim In the above scenario Cloudlets are used for creation of jobs in the cloud environment once the jobs are created it must be allocate to the datacenter. In the datacenter depending upon the number of jobs the datacenter are allocated in the next step the ABC Scheduling algorithm is applied after implementing the scheduling algorithm the scheduled task are assigned to the virtual machine after the scheduling process the final result which obtained will be Minimum task finishing time, Mean task finishing time and Minimum load balancing time. 


\section{TASK SCHEDULING IN CLOUD COMPUTING USING ABC ALGORITHM}

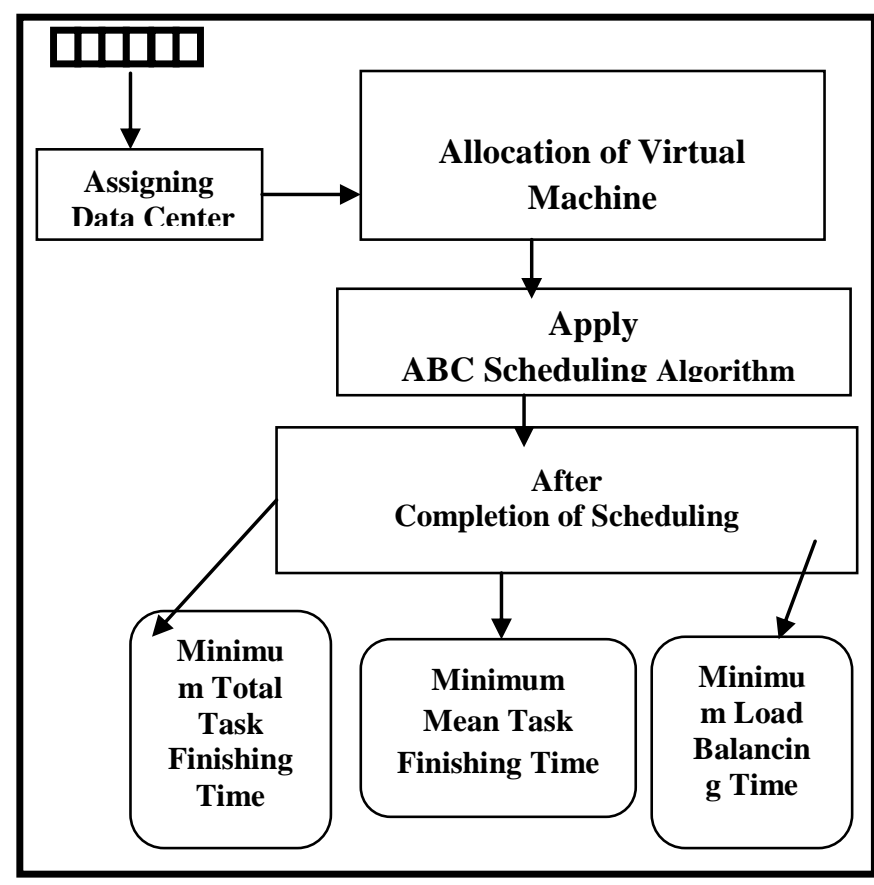

Fig: 4.1 over all Architecture Diagram

\subsection{Algorithm Used}

The goal of our task scheduling is used to make task scheduled reasonably by using this algorithm we are directly concentrate on the shortest finishing time of jobs such as Mean task finishing time, total finishing time and load balancing time

The following steps are used in artificial bee colony scheduling algorithm

1. Get the status of the current machine

2. Allocate the job to the datacenter

3. Applying the $\mathrm{ABC}$ algorithm with QoS

4. Calculate the Mean finishing time, Total finishing time and load balancing time.

\subsubsection{Current status of the machine}

The first step is to check whether the machine is free or full of activity with another job if the machine is free then the current machine list with the job list is assigned. In order to increase the current list we can append both machine and jobs

\subsubsection{Allocation of Jobs in Datacenter}

In this step the jobs are allocated to the datacenter, depending on the size of the job the data centers are allocated.

\subsection{Applying the $A B C$ algorithm}

In this step we are applying the artificial bee colony algorithm $(\mathrm{ABC})$ with the help of virtual machine the task are scheduled and the QoS parameters time is mainly used. In order scheduling the task the following steps are done.

\subsubsection{Data center creation}

Step 1: start

Step 2: Set the characteristics

Step 3: set the virtual machine allocation policy in it

Step 4: set the last process time of data center

Step 5: set storage list, vm list, debt list

\subsubsection{Vm description}

Step 1: start

Step 2: Set the MIPS //int []$=\{50.100,150,200\}$

Step 3: set the number of processing element

Step 4: Create the virtual machine with broker id, average MIPS, Processing element, ram, bandwidth and dynamic workload as input

\subsubsection{Creation of Cloudlet}

Step 1: long length $=c$; // 10 min on 250 MIPS

Step 2: set the MIPS

Step 3: set the processing element number, file size, output size

Step4: Create cloudlet=new cloudlet (i,c, pes Number, file size, output Size new utilization model ( );

\subsubsection{Cloud Coordinator}

Step 1: start

Step 2: Set the cloud machine Properties.

Step 3: for each job set the job id, starting time, datacenter, virtual machine

Step 4: By using the length calculate list of jobs using round robin fashion and execute the selected jobs

\subsubsection{Cloud Scheduler}

Step 1: start

Step 2: Creates a new Cloudlet Scheduler object.

Step 3: Receives a cloudlet to be executed in the VM managed by this scheduler

Step 4: Processes a finished cloudlet and gets the status of a cloudlet.

Step 5: Return status of the cloudlet, -1 if cloudlet not found.

Step 6: Stop 


\subsubsection{Data center Character}

Step 1: start

Step 2: The Datacenter resource are created

Step 3: Allocates a new Datacenter Characteristics object

Step 4: Gets a Machine with at least one empty Process Elements

Step 5: Gets the total MIPS rating, which is the sum of MIPS rating of all machines in resource

\subsubsection{Vm allocation policy}

Step 1: start

Step 2: Allocates a new Vm Allocation Policy object.

Step 3: Allocates a specified host for a given VM.

Step 4: Optimize allocation of the VMs according to current utilization.

Step 5: return the array list $<$ hash map $<$ string, object $>>$

Step 6: Stop

\section{3 calculate the mean finishing time, total finishing and load balancing time}

After scheduling the task by using the $\mathrm{ABC}$ scheduling algorithm the total finishing time, mean finishing time, load balancing time is calculated and the resulting time is compared with the existing result of ant colony optimization algorithm.

\section{EXPERIMENTAL RESULTS AND PERFORMANCE EVALUATION}

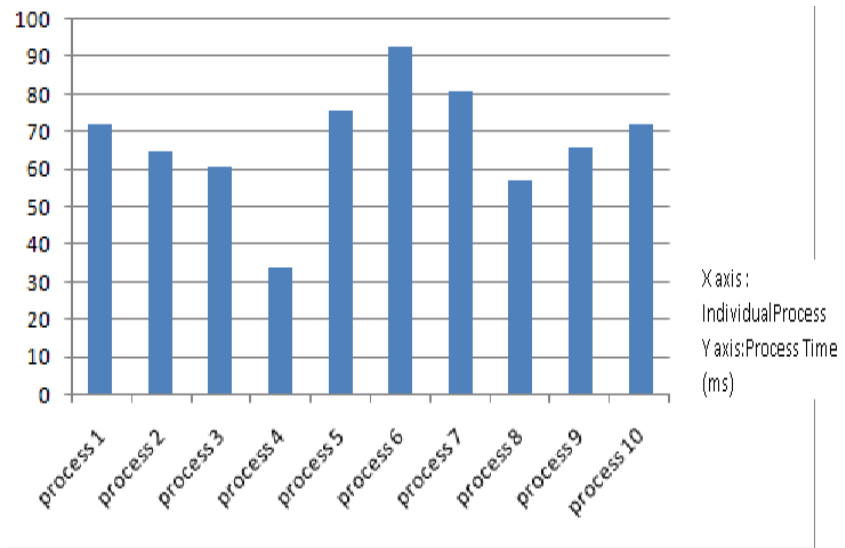

\begin{tabular}{|c|c|c|c|c|c|c|c|c|c|c|}
\hline Process & p1 & $\begin{array}{c}\text { p } \\
2\end{array}$ & p3 & p4 & p5 & p6 & p7 & p8 & p9 & p10 \\
\hline $\begin{array}{c}\text { Processing } \\
\text { time }\end{array}$ & 72 & 65 & 61 & 34 & 76 & 93 & 81 & 57 & 66 & 72 \\
\hline
\end{tabular}

Fig: 5.1 Graph for Individual task finishing Time using Ant colony Algorithm

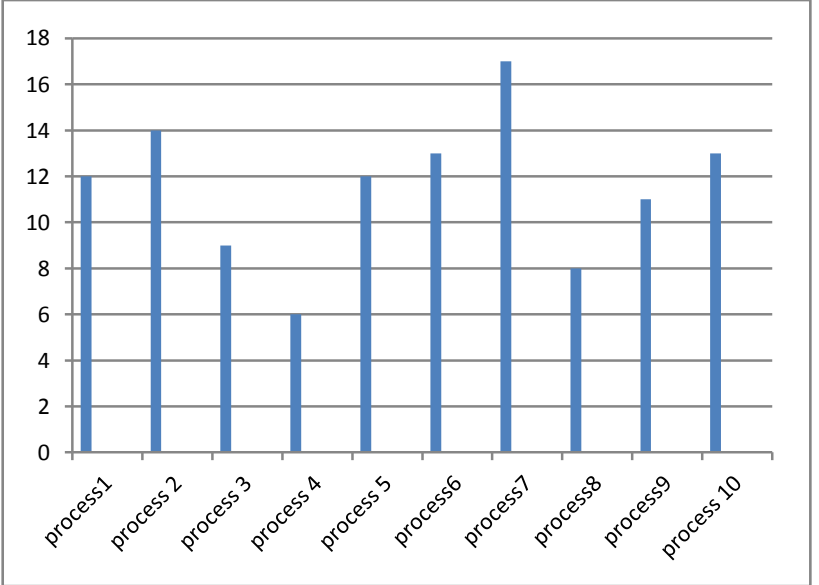

\begin{tabular}{|c|c|c|c|c|c|c|c|c|c|c|}
\hline Process & p1 & $\begin{array}{c}\text { p } \\
2\end{array}$ & p3 & p4 & p5 & p6 & p7 & p8 & p9 & p10 \\
\hline $\begin{array}{c}\text { processing } \\
\text { time }\end{array}$ & 10 & 12 & 08 & 07 & 13 & 12 & 16 & 09 & 11 & 14 \\
\hline
\end{tabular}

Fig: 5.2 Graph for Individual task finishing Time for Artificial bee colony Algorithm

After the Implementation of the system the required simulations are performed using cloudsim Simulator. The above graph depicts the Simulation time Total Task finishing Time. In this graph the $\mathrm{X}$-axis for the total numbers of process and the $\mathrm{Y}$-axis the Task finishing time for the allocated graph for each and every process.

\section{CONCLUSION AND FUTURE ENHANCEMENTS}

In this research work the $\mathrm{ABC}$ algorithm is used to schedule the tasks and their task assignments. Through its evolutionary its process the Algorithm produce the best total task finishing time, Mean task finishing time and Load balancing time possible which is previously made the cloudsim simulator is used to simulate the results the comparative study of the different task Scheduling algorithm is performed. The study of cloudsim is used to understand the creation of cloud environment in simulation through the java programming language In future enhancement the efficiency of the optimization algorithm is increased by improving the datacenter efficiency with adding more parameters. If these assignments are done then automatically the scheduling time is reduced and the best result is obtained. This can be an innovative future enhancement for the system.

\section{REFERENCES}

[1] AChin Soon Chong.S (2009) "A Bee Colony Optimization Algorithm to JobShop Scheduling", Proceedings of the 2006 Winter Simulation Conference.

[2] AHorst.Wedde, Muddassar Farooq, and Yue Zhang (2009)"Beehive: An Efficient Fault- Tolerant Routing Algorithm Inspired by Honey Bee Behavior", ANTS 2004, LNCS 3172, pp. 83-94, 2004. cSpringer-Verlag Berlin Heidelberg 2004

[3] Bitam Salim .G (2010) "Bees Life Algorithm for Job Scheduling in CloudComputing", BEES 2010, LNCS 5612, pp. 22-54, 212. ICCIT - 2012 
[4] Jingpeng Li A Bayesian (2003) "Optimization Algorithm for the Nurse Scheduling Problem" Proceedings of 2003 Congress on Evolutionary Computation (CEC2003), pp. 2149-2156, IEEE Press, Canberra, Australia, 2003.

[5] Nadezda Stanarevic Milan Tuba, and Nebojsa Bacanin (2011) "Modified Artificial bee colony algorithm for constrained problems optimization"International Journal of Mathematical Models and Methods in Applied Sciences

[6] Nidhal Kamel Taha El-Omari (2010) "Developing Optimization Algorithm Using Artificial Bee Colony System" Ubiquitous Computing and Communication Journal.

[7] Pan.Q, Li.H, Xie.S, Wang.S (2010) "A Hybrid Artificial Bee Colony Algorithm for FlexibleJobShop Scheduling Problems", Int. J. of Computers, Communications \&
Control, ISSN 1841-9836, E-ISSN 1841-9844 Vol. VI(2011), No. 2 (June), pp. 286-296

[8] Qi Cao, Zhi-Bo Wei and Wen-Mao Gong (2009)"An Optimized Algorithm For Task Scheduling Based On Activity Based CostinginCloud Computing", $3^{\text {rd }}$ International Conference on Bioinformatics and Biomedical Engineering, (ICBBE)

[9] Rodrigo.W Calheiros et.al (2010) Cloudsim: "A Novel Framework Modeling and Simulation of Cloud Computing Infrastructures and Services" 5th International Conference on Simulation and networking 2010.

[10] Wuqi Gao and Fengju Kang (2012) "Cloud Simulation Resource Scheduling Algorithm Based Multidimension Quality of Service Information Technology Journal Year 2012 Volume: 11. 